\title{
Scapholunate Ligament Internal Brace 360-Degree Tenodesis (SLITT) Procedure
}

\author{
Sanjeev Kakar, MD, FAOA ${ }^{1}$ Ryan M. Greene, DO, MS ${ }^{1}$ \\ ${ }^{1}$ Department of Orthopedic Surgery, Mayo Clinic, Rochester, \\ Minnesota \\ J Wrist Surg 2018;7:336-340. \\ Address for correspondence Sanjeev Kakar, MD, FAOA, Department \\ of Orthopedic Surgery, Mayo Clinic, 200 First Street SW, Rochester, \\ MN 55902 (e-mail: Kakar.Sanjeev@mayo.edu).
}

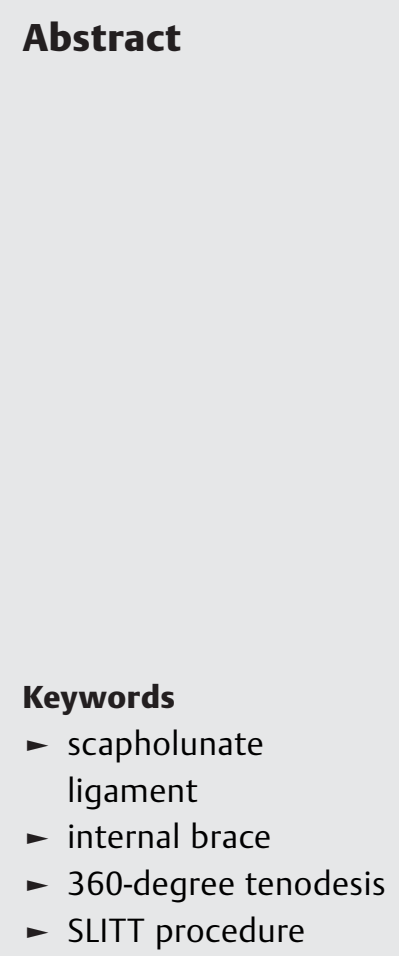

Background Scapholunate (SL) joint instability is one of the most common injuries of the wrist and may result from a fall or high-energy mechanism on the outstretched hand. The purpose of this case report is to describe the outcome of a 360-degree tenodesis to the SL joint with an internal brace (SLITT) for the treatment of SL instability.

Case Description A 42-year-old male patient underwent SL ligament reconstruction with the SLITT procedure 12 months after injury. Given the intrinsic stability of the reconstruction, Kirschner $(\mathrm{K})$ wires were not used and an early range of motion protocol was initiated. Thirteen months after his reconstruction, he was back at work with maintenance of his carpal alignment.

Discussion Since its initial description, a myriad of different surgical techniques for $\mathrm{SL}$ instability have been devised with varied success. These include capsular shrinkage, dorsal capsulodesis, reduction-association with a screw of the scapholunate joint (RASL), scapholunate axis method (SLAM), bone ligament bone grafts, and a variety of tendon reconstructions. Possible explanations for this varied outcome may be related to the use of soft tissue reconstructions for irreducible injuries and reconstruction of only the dorsal SL ligament. In addition, many of these techniques involve prolonged immobilization with the use of K-wires.

Clinical Relevance The SLITT procedure reconstructs both the volar and dorsal SL ligament. Given the added stability afforded by intrinsic bracing, we feel that this reconstruction may permit earlier range of motion without the need for K-wire stabilization.
Scapholunate (SL) joint instability is one of the most common injuries of the wrist and may result from a fall or high-energy mechanism on the outstretched hand. If unrecognized, the injury may lead to functional impairment and posttraumatic arthritis. $^{1-3}$

Since its initial description, ${ }^{4}$ a myriad of different surgical techniques have been devised with varied success. These include capsular shrinkage, dorsal capsulodesis, reductionassociation with a screw of the scapholunate joint (RASL), scapholunate axis method (SLAM), bone ligament bone grafts and a variety of tendon reconstructions. ${ }^{5-12}$ Many of these constructs require prolonged immobilization with Kirschner (K) wire stabilization, which may break, become infected, or

received

July 10, 2017

accepted after revision

December 26, 2017

published online

January 30, 2018 require a secondary procedure for its removal. In addition, the outcomes of SL reconstructions are unpredictable. ${ }^{1,13,14}$ Possible explanations for this may be related to the use of soft tissue reconstructions for irreducible injuries and reconstruction of only the dorsal SL ligament. Indeed, the sectioning studies by Berger ${ }^{15}$ noted that while the dorsal SL ligament has a yield strength of close to $300 \mathrm{~N}$, the palmar region provides $120 \mathrm{~N}$ of breaking strength.

To try and correct the torsional instability that may result from dorsal only repairs, techniques have been described that address both dorsal and volar SL ligaments. Henry ${ }^{16}$ reconstructed both the volar and dorsal SL ligaments using the flexor carpi radialis (FCR) and noted an excellent result at
Copyright $\odot 2018$ by Thieme Medical Publishers, Inc., 333 Seventh Avenue, New York, NY 10001, USA. Tel: +1(212) 584-4662.
DOI https://doi.org/ 10.1055/s-0038-1625954. ISSN 2163-3916. 
8 years postsurgery. Chee etal ${ }^{17}$ reported on the antipronation tenodesis using a circumferential wrap of the FCR to extend the scaphoid, supinate the distal carpal row, and resist triquetral extension. In patients treated for SL dissociation, they returned to their normative condition with grip strength of $70 \%$ of the contralateral side.

The following case report describes the result of a SL ligament reconstruction using a 360-degree tenodesis augmented with an internal brace.

\section{Case Report}

A 42-year-old, right-hand dominant warehouse male worker presented 1 year following a work-related injury where he sustained a twisting injury to his left wrist in a conveyor belt. He had undergone radiocarpal joint arthroscopy at an outside institution and an ulnotriquetral ligament repair. Given his continued pain, he was seen in our clinic with dorsal radial wrist pain. Examination demonstrated tenderness over the SL interval with a negative Watson shift test. ${ }^{18}$ He had reduced range of motion, and his grip strength was $46 \%$ of the uninjured wrist (28 vs. $60 \mathrm{~kg}$ ). Plain radiographs demonstrated no increase in diastasis on the clenched fist view. A MRI demonstrated an occult ganglion overlying the dorsal aspect of the SL ligament as well as a possible SL tear. The patient underwent ultrasound-guided aspiration of his cyst and a period of immobilization. Despite this, he continued to have pain over his SL ligament and was unable to work. As such, he underwent wrist arthroscopy for which midcarpal examination demonstrated Geissler ${ }^{19}$ IV SL diastasis with a step-off and a positive drive-through sign ( - Fig. 1). Given the chronicity of the injury, we proceeded with a SL ligament intrinsic brace 360-degree tenodesis (SLITT) reconstruction.

A longitudinal incision was fashioned over the dorsum of the wrist. The EPL tendon was identified, and the third extensor compartment released. The second as well as the fourth and

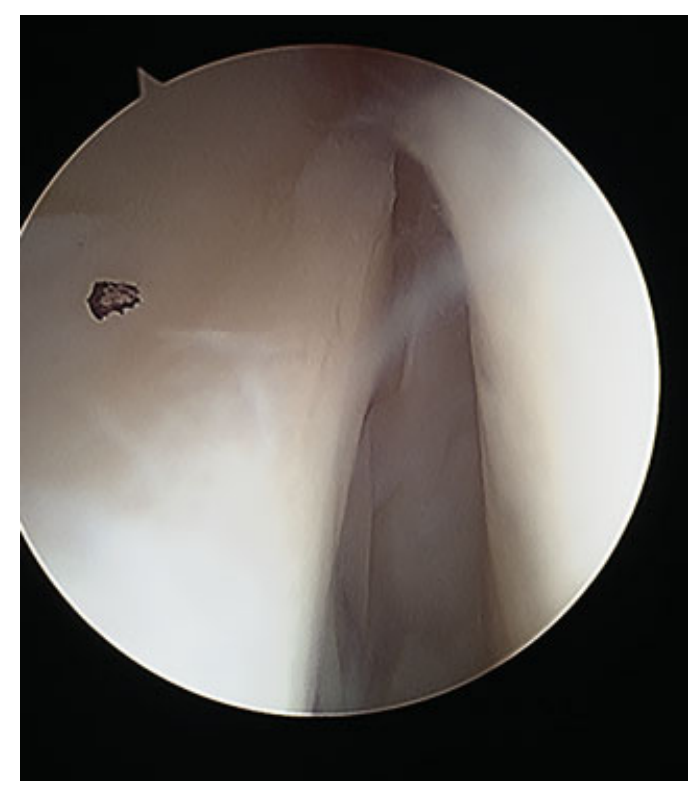

Fig. 1 Midcarpal arthroscopy demonstrating type 4 scapholunate instability. fifth extensor compartments were opened with radial- and ulnar-based extensor retinacular flaps, respectively. A posterior interosseous neurectomy was performed given an encountered neuroma. After a ligament-sparing capsulotomy, ${ }^{20}$ examination of the carpus confirmed a reducible SL dissociation (-Fig. 2). Given this, attention was diverted palmarly where an extended carpal tunnel release was performed and the palmaris longus harvested and trimmed so that it was $2.5 \mathrm{~mm}$ wide. To correct any dorsal intercalated segment instability of the carpus, $0.045 \mathrm{~K}$-wires were placed in the central aspect of the scaphoid (dorsal distal to palmar proximal) and central lunate (dorsal proximal to palmar distal) and confirmed on fluoroscopic imaging. In neutral alignment of the scaphoid and the lunate, the K-wires were placed parallel to each other in a dorsal to volar direction. A 3-mm drill hole was made in the lunate and a $2.5-\mathrm{mm}$ hole made within the scaphoid to permit a single passage of the graft through the scaphoid and a double passage through the lunate to complete the 360-degree tenodesis. To assist with the reduction of the SL interval, a K-wire was placed in the distal scaphoid and another within the triquetrum and a reduction clamp placed. The autograft was whip stitched at each end with 4-0 fiber loop and then passed via a tendon passer from dorsal to volar through the lunate, volar to dorsal through the scaphoid, and dorsal to volar through the lunate (-Figs. 3 and $\mathbf{4}$ ). The graft was tensioned and the wrist taken through passive range of motion to decrease its creep. The graft was secured within the scaphoid and lunate with $3 \times 8 \mathrm{~mm}$ biotenodesis screws (Arthrex, Naples, FL) placed from dorsal to volar. Through the tenodesis screw holes, a $1.3-\mathrm{mm}$ diameter suture tape was passed from dorsal to palmar through the scaphoid and the lunate and tied palmarly (-Figs. 5 and $\mathbf{6}$ ). By passage through the screws, this prevents the internal brace from cutting out through the carpus. All K-wires were removed. To control flexion of the scaphoid, the dorsal limb of the tendon graft can be tethered to the distal pole of the scaphoid. The capsule

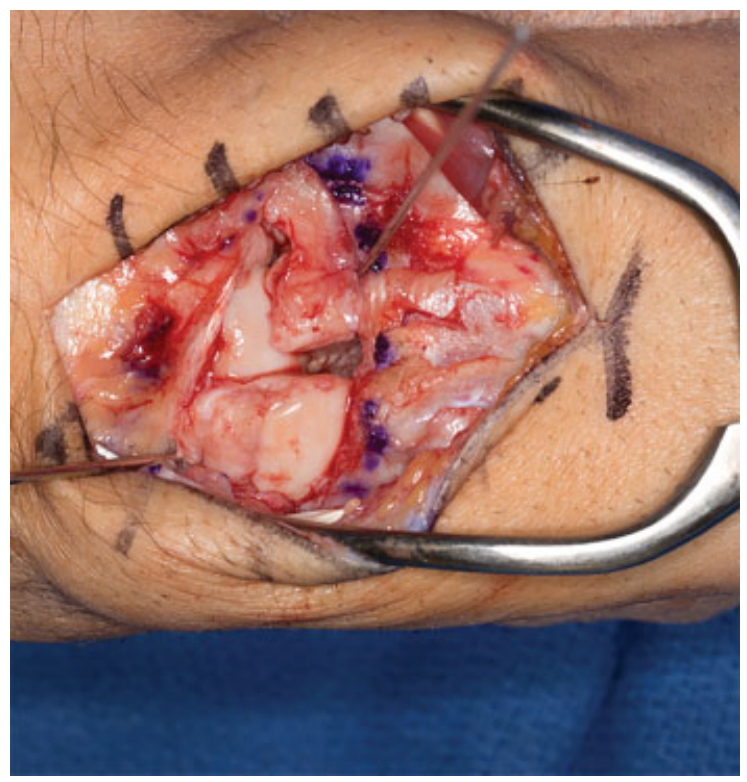

Fig. 2 Intraoperative photograph demonstrating $0.045 \mathrm{~K}$-wire placement to determine reducibility of the scapholunate joint. 


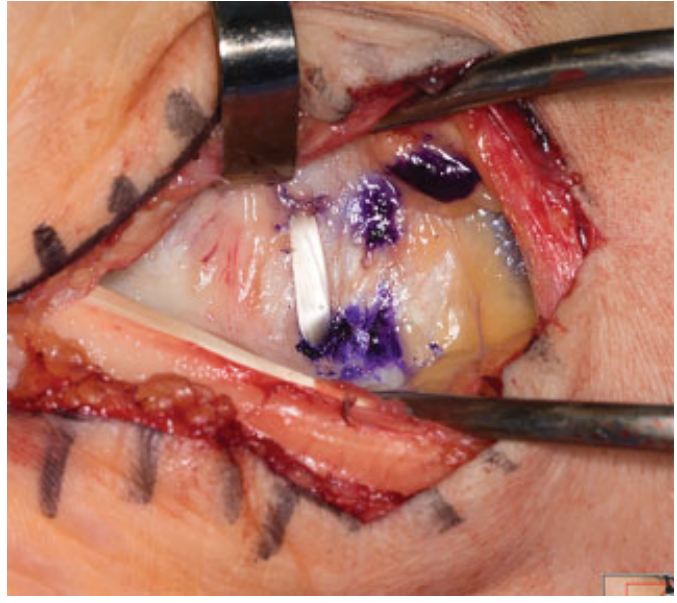

Fig. 3 Reconstruction of the volar scapholunate ligament.

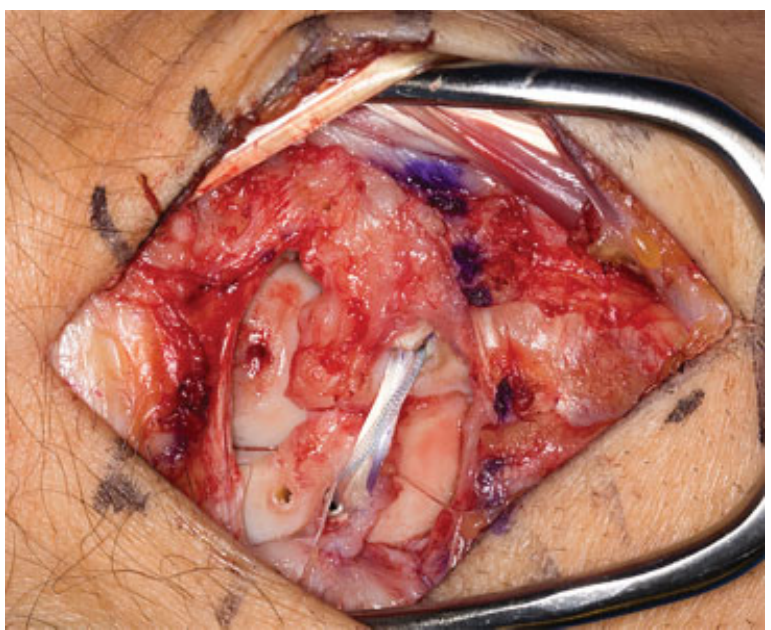

Fig. 5 Dorsal scapholunate ligament reconstruction with suture tape intrinsic brace.

was closed with 2-0 nonabsorbable suture. The patient was immobilized in a short arm cast for 4 weeks after which he began dart thrower's wrist range of motion and flexor carpi radialis and extensor carpi radialis brevis strengthening.

At 13 months postoperatively, the patient was happy and back at work. His pain was zero at rest and less than 4 out of 10 on a visual analog scale (VAS) with maximal effort. His grip strength of the affected wrist continued to improve and was $66 \%$ ( $25 \mathrm{vs.} 38 \mathrm{~kg}$ ) of contralateral side compared with $46 \%$ preoperatively with symmetrical range of motion ( - Fig. 7).

\section{Discussion}

The treatment of scapholunate instability continues to vex surgeons. The injury pattern ranges from a minor sprain to posttraumatic arthritis. Many techniques have been proposed and are based upon controlling the scaphoid that assumes a position of flexion and pronation. These range from dorsal capsulodesis to reduction and association of the scaphoid and lunate, modified Brunelli procedure to the

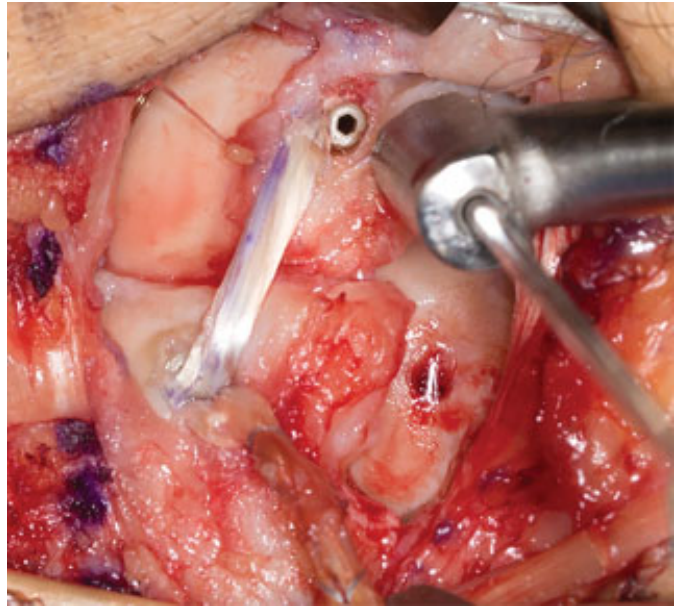

Fig. 4 Reconstruction of the dorsal scapholunate ligament. Note the cannulation within the biotenodesis screws for internal brace passage.

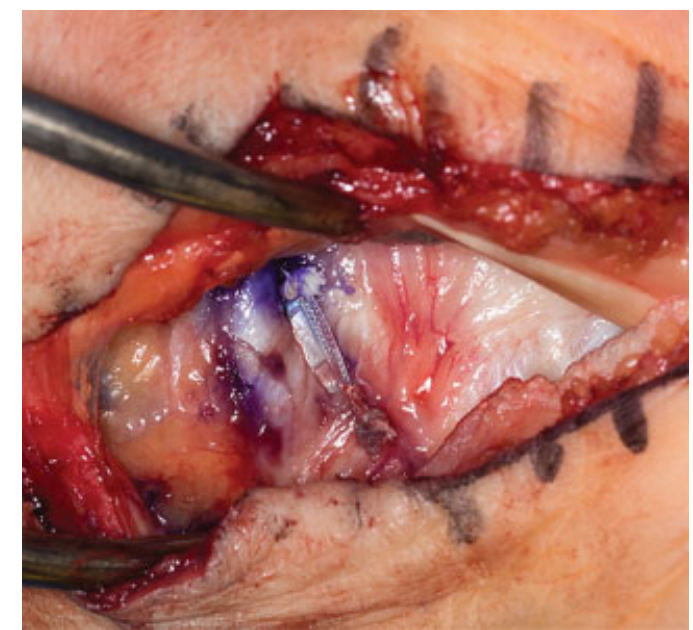

Fig. 6 Volar scapholunate ligament reconstruction with suture tape intrinsic brace.

SLAM reconstruction. Many procedures have proven technically demanding with varied results. ${ }^{1-3,5,6,8,10,15-17,21-26}$

Berger's detailed anatomic prosections noted that the SL ligament comprised a dorsal, membranous and volar region. The palmar ligament had a yield strength of $120 \mathrm{~N}$ compared with that of the dorsal ligament which exhibited a breaking strength of $300 \mathrm{~N}$. Given this, traditional techniques have concentrated on reconstructing the dorsal ligament only. Garcia-Elias ${ }^{1}$ et al reported on the outcomes of the triligament tenodesis technique in 38 patients at an average follow-up of 46 months. Twenty-eight patients reported no pain, and 29 returned to their original occupation. Of note, $18 \%$ of patients developed mild signs of arthritis. Patients were immobilized for a prolonged period of time and required a secondary procedure for K-wire removal at an average of 8 weeks postoperatively. Talwalkar et $\mathrm{al}^{27}$ reported on 162 patients at an average of 4 years following modified triligament tenodesis. Seventy-seven (62\%) patients reported minimal or no pain at time of last examination, and there was no difference observed in the outcomes between static or dynamic SLIL instability. 


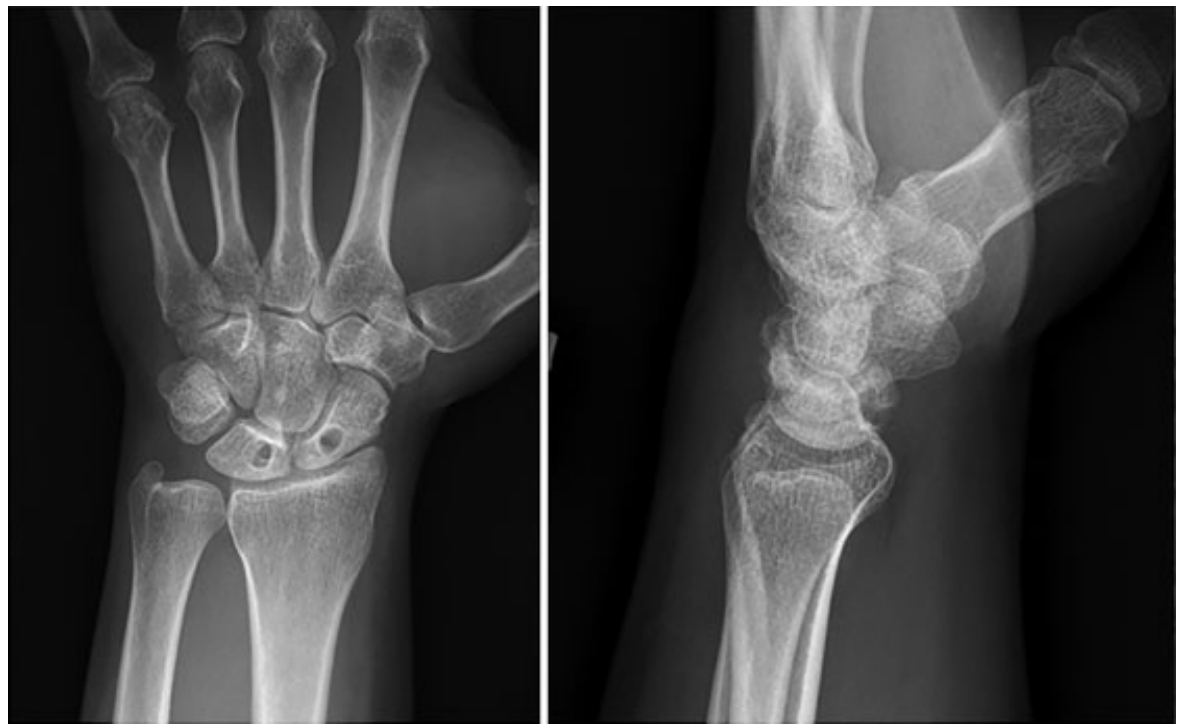

Fig. 7 Posteroanterior and lateral radiographs of the patient 13 months postsurgery.

As load is transferred across the SL joint, the morphology of the SL ligament may be important to resist torsional and translatory moments. Indeed, part of the reason why there may be unpredictability in dorsal ligament reconstruction techniques is the failure to repair the volar ligament. Albeit not as strong as the dorsal segment, it may aid in resisting rotation across the joint. ${ }^{15}$ As such, there has been an increasing interest to address the volar SL such as a volar capsulodesis $^{28}$ to circumferential grafts around the scaphoid and lunate. ${ }^{29}$ As reported by Chee et al, ${ }^{17}$ a strip of the FCR tendon can be passed from volar to dorsal through the scaphoid and dorsal to volar through the triquetrum in an antipronation tenodesis to correct carpal malalignment. Ho et al described an arthroscopy-assisted technique of reconstructing the volar and dorsal SL ligaments using a palmaris longus graft. Seven- teen patients with chronic SL instability were treated and followed on average for 48 months. Eleven of 17 patients reported no pain, the average SL gap was $2.9 \mathrm{~mm}$, and 13 patients returned to their preinjury job level. There was one case of scaphoid ischemia that did not progress or become symptomatic. We did not notice any avascular necrosis within the series presented but vigilance needs to be paid. Similar results have been reported by Henry following volar and dorsal SLIL repair with immobilization for 10 to 12 weeks. ${ }^{16}$ Pin removal occurred at 8 weeks postoperatively.

For the RASL procedure, Larson and Stern ${ }^{30}$ described results of intervention in eight wrists with a mean follow-up of 38 months. Radiographic success was described in $3 / 8$ wrists with $5 / 8$ demonstrating radiographic failure at approximately 5 months postoperatively. All patients followed a postoperative
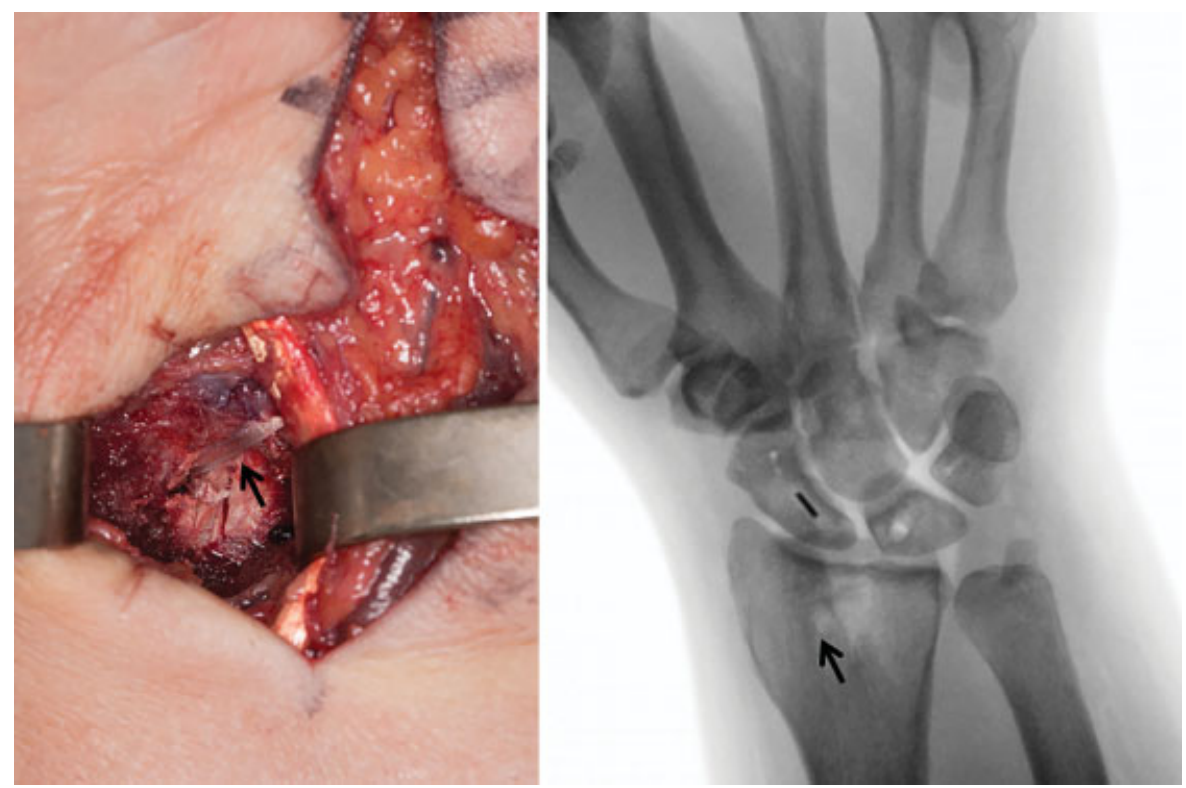

Fig. 8 Intraoperative photo and radiograph demonstrating recreation of the long radiolunate ligament (arrow denotes recreation of long radiolunate ligament). 
protocol that included cast immobilization of approximately 6 weeks with strengthening programs initiated at 10 to 12 weeks. Mathoulin et $\mathrm{al}^{31}$ described results of arthroscopic dorsal capsuloligamentous repair with mean follow-up of 11.4 months in 36 patients. Patients were immobilized with a cast for 8 weeks and K-wires were removed ( 16 cases) and physical therapy initiated. Patient grip strength on average increased to $92 \%$ of unaffected side with significant reduction in VAS score (mean VAS score $=0.5 / 10$ ).

The concept of the SLITT procedure is to provide resistance to load along multiple axial planes and prevent scaphoid flexion with the distal tether. With the addition of an internal brace, it affords immediate construct stability, thereby obviating the need for K-wire stabilization and prolonged immobilization. Given this inherent stability, it may permit earlier range of motion in patients without the need for secondary procedures to remove K-wires. Indeed as shown in the case example, Kwires were not used, wrist motion began at 4 weeks after surgery, and the SL gap was maintained at follow-up. Its main indication is for reducible Taleisnik ${ }^{11}$ type I SL instability. In cases where there is ulnar translocation of the lunate (Taleisnik type 2), the long radiolunate ligament can be reconstructed using the volar tail of the tendon graft and internal brace being tethered to the volar distal radius (-Fig. 8).

The SL ligament internal brace 360-degree tenodesis procedure presented in this article aims to address the multiplanar instability that exists after SL injury as well as permitting immediate construct stability that replicates native SL yield strength. The augmentation with the internal brace adds several advantages over tendon grafts alone and is better suited to resist immediate loads and diastasis of the SL joint and obviates the need for K-wire stabilization.

\section{Conflict of Interest}

Dr. Kakar is a Consultant for Arthrex Inc. but receives no royalties or payments for this technique or article. All the other authors report no conflict of interest.

\section{References}

1 Garcia-Elias M, Lluch AL, Stanley JK. Three-ligament tenodesis for the treatment of scapholunate dissociation: indications and surgical technique. J Hand Surg Am 2006;31(01):125-134

2 Rainbow MJ, Wolff AL, Crisco JJ, Wolfe SW. Functional kinematics of the wrist. J Hand Surg Eur Vol 2016;41(01):7-21

3 Pomerance J. Outcome after repair of the scapholunate interosseous ligament and dorsal capsulodesis for dynamic scapholunate instability due to trauma. J Hand Surg Am 2006;31(08):1380-1386

4 Linscheid RL, Dobyns JH, Beabout JW, Bryan RS. Traumatic instability of the wrist. Diagnosis, classification, and pathomechanics. J Bone Joint Surg Am 1972;54(08):1612-1632

5 Darlis NA, Kaufmann RA, Giannoulis F, Sotereanos DG. Arthroscopic debridement and closed pinning for chronic dynamic scapholunate instability. J Hand Surg Am 2006;31(03):418-424

6 Darlis NA, Weiser RW, Sotereanos DG. Partial scapholunate ligament injuries treated with arthroscopic debridement and thermal shrinkage. J Hand Surg Am 2005;30(05):908-914

7 Blatt G. Capsulodesis in reconstructive hand surgery. Dorsal capsulodesis for the unstable scaphoid and volar capsulodesis following excision of the distal ulna. Hand Clin 1987;3(01): 81-102

8 Moran SL, Cooney WP, Berger RA, Strickland J. Capsulodesis for the treatment of chronic scapholunate instability. J Hand Surg Am 2005;30(01):16-23

9 Linscheid RL, Dobyns JH. Treatment of scapholunate dissociation. Rotatory subluxation of the scaphoid. Hand Clin 1992;8(04):645-652

10 Brunelli GA, Brunelli GR. A new technique to correct carpal instability with scaphoid rotary subluxation: a preliminary report. J Hand Surg Am 1995;20(3 Pt 2):S82-S85

11 Taleisnik J. The wrist. New York, NY: Churchill Livingstone; 1985

12 Van Den Abbeele KL, Loh YC, Stanley JK, Trail IA. Early results of a modified Brunelli procedure for scapholunate instability. J Hand Surg [Br] 1998;23(02):258-261

13 Szabo RM. Scapholunate ligament repair with capsulodesis reinforcement. J Hand Surg Am 2008;33(09):1645-1654

14 Weiss AP. Scapholunate ligament reconstruction using a boneretinaculum-bone autograft.J Hand Surg Am 1998;23(02):205-215

15 Berger RA. The gross and histologic anatomy of the scapholunate interosseous ligament. J Hand Surg Am 1996;21(02):170-178

16 Henry M. Reconstruction of both volar and dorsal limbs of the scapholunate interosseous ligament. J Hand Surg Am 2013;38 (08):1625-1634

17 Chee KG, Chin AY, Chew EM, Garcia-Elias M. Antipronation spiral tenodesis-a surgical technique for the treatment of perilunate instability. J Hand Surg Am 2012;37(12):2611-2618

18 Watson HK, Ashmead D IV, Makhlouf MV. Examination of the scaphoid. J Hand Surg Am 1988;13(05):657-660

19 Geissler WB. Arthroscopic management of scapholunate instability. J Wrist Surg 2013;2(02):129-135

20 Berger RA, Bishop AT. A fiber-splitting capsulotomy technique for dorsal exposure of the wrist. Tech Hand Up Extrem Surg 1997;1 (01):2-10

21 Aviles AJ, Lee SK, Hausman MR. Arthroscopic reduction-association of the scapholunate. Arthroscopy 2007;23(01):105.e1-105.e5

22 Yao J, Zlotolow DA, Lee SK. ScaphoLunate axis method. J Wrist Surg 2016;5(01):59-66

23 Short WH, Werner FW, Green JK, Masaoka S. Biomechanical evaluation of the ligamentous stabilizers of the scaphoid and lunate: Part II. J Hand Surg Am 2005;30(01):24-34

24 Kitay A, Wolfe SW. Scapholunate instability: current concepts in diagnosis and management. J Hand Surg Am 2012;37(10): 2175-2196

25 Berger RA, Imeada T, Berglund L, An KN. Constraint and material properties of the subregions of the scapholunate interosseous ligament. J Hand Surg Am 1999;24(05):953-962

26 Berger RA, Blair WF, Crowninshield RD, Flatt AE. The scapholunate ligament. J Hand Surg Am 1982;7(01):87-91

27 Talwalkar SC, Edwards AT, Hayton MJ, Stilwell JH, Trail IA, Stanley JK. Results of tri-ligament tenodesis: a modified Brunelli procedure in the management of scapholunate instability. J Hand Surg [Br] 2006;31(01):110-117

28 van Kampen RJ, Bayne CO, Moran SL. A new technique for volar capsulodesis for isolated palmar scapholunate interosseous ligament injuries: a cadaveric study and case report. J Wrist Surg 2015;4(04):239-245

29 Ho PC, Wong CW, Tse WL. Arthroscopic-assisted combined dorsal and volar scapholunate ligament reconstruction with tendon graft for chronic SL instability. J Wrist Surg 2015;4(04):252-263

30 Larson TB, Stern PJ. Reduction and association of the scaphoid and lunate procedure: short-term clinical and radiographic outcomes. J Hand Surg Am 2014;39(11):2168-2174

31 Mathoulin CL, Dauphin N, Wahegaonkar AL. Arthroscopic dorsal capsuloligamentous repair in chronic scapholunate ligament tears. Hand Clin 2011;27(04):563-572 